\title{
Comparison of Depth Dose Distributions Using Cerenkov Fiber-Optic Dosimeter and Monte Carlo Simulation for HDR Brachytherapy
}

\author{
Sang Hun Shin', Young Beom Song², Mingeon Kim², Hye Jin Kim², Wook Jae Yoo', \\ Kyoung Won Jang1, Bongsoo Lee ${ }^{2 *}$ \\ ${ }^{1}$ School of Biomedical Engineering, BK21 Plus Research Institute of Biomedical Engineering, \\ Konkuk University, Chungju-si, Korea \\ ${ }^{2}$ School of Energy Systems Engineering, Chung-Ang University, Seoul, Korea \\ Email: "bslee@cau.ac.kr
}

Received 14 July 2016; accepted 8 August 2016; published 15 August 2016

\begin{abstract}
In this study, we fabricated a Cerenkov fiber-optic dosimeter (CFOD) without any scintillator to measure Cerenkov radiation signals owing to gamma-rays. The relative depth dose (RDD) distributions of Ir-192 HDR brachytherapy source were obtained by using the CFOD based on a subtraction method and the RDD curve was compared with the simulation result of Monte Carlo N-particle extended transport code (MCNPX). Finally, we demonstrated that the CFOD can be used to measure real-time dose information for HDR brachytherapy.
\end{abstract}

\section{Keywords}

Cerenkov Fiber-Optic Dosimeter, Cerenkov Radiation, Gamma-Ray, Relative Depth Dose, HDR Brachytherapy

\section{Introduction}

In general, high-energy charged particles can produce Cerenkov radiation or light in various kinds of transparent materials [1]. In high-dose-rate (HDR) brachytherapy applications that use various radiation sources with intermediate energies, in the hundred $\mathrm{keV}$ range, Cerenkov radiation can be also generated. This Cerenkov radiation is well known as an additional signal for the fiber-optic dosimeters (FODs) using scintillators and thus, it can interfere with the accurate measurement of the dose information as an unwanted light signal. However, Cerenkov light can be of utility for dosimetry because it is one of the various light signals generated from the sensing areas of FODs by the radiation interactions. Radiation-induced light signals including Cerenkov light can be obtained simply using the optical fibers without any scintillating material [2]-[8].

*Corresponding author.

How to cite this paper: Shin, S.H., Song, Y.B., Kim, M., Kim, H.J., Yoo, W.J., Jang, K.W. and Lee, B. (2016) Comparison of Depth Dose Distributions Using Cerenkov Fiber-Optic Dosimeter and Monte Carlo Simulation for HDR Brachytherapy. Journal of Applied Mathematics and Physics, 4, 1499-1502. http://dx.doi.org/10.4236/jamp.2016.48157 
In this study, we fabricated a novel Cerenkov fiber-optic dosimeter (CFOD) using plastic optical fibers (POFs), a photomultiplier tube (PMT; H12525-01, Hamamatsu Photonics), and a photon-counting unit (C8855-01, Hamamatsu Photonics) to measure the light intensities of Cerenkov radiation induced by gamma-rays. Using the CFOD, the relative depth dose (RDD) distributions of a HDR brachytherapy source were measured and then, the RDD curve was compared with simulation result of Monte Carlo N-particle extended transport code (MCNPX).

\section{Materials and Methods}

The sensing probe of the CFOD is composed of two identical POFs with different lengths to measure Cerenkov radiation [9]. The length difference of two POFs (i.e., probes 1 and 2) is $10 \mathrm{~mm}$ to apply the subtraction method, as shown in Figure 1. In general, the subtraction method can be employed for measuring the difference of interest between two sensor signals [10]. In this study, we used two step-index multimode POFs (GH-4002 Mitsubishi Rayon) to generate and transmit Cerenkov radiation signals owing to the gamma-rays emitted from a HDR brachytherapy source. The core material of the POF is polymethylmethacrylate (PMMA) and the cladding layer is made of fluorinated polymer. The refractive indices of the core and the cladding at the design wavelength of $650 \mathrm{~nm}$ are 1.49 and 1.402, respectively; thereby the NA is about 0.5 . And, the core diameter is $0.98 \mathrm{~mm}$ and the cladding thickness is $0.01 \mathrm{~mm}$. The maximum transmission loss of the POF is $190 \mathrm{~dB} / \mathrm{km}$ when used with 650 nm collimated light.

Ir-192 with a radioactivity of $8.14 \mathrm{Ci}$ is used as a HDR brachytherapy source. The diameter and the length of Ir-192 are 0.6 and $3.5 \mathrm{~mm}$, respectively. Ir-192 emits gamma-rays with the energy range from 50 to $800 \mathrm{keV}$ and the energy peaks at 316 and $468 \mathrm{keV}$. The average energy of the gamma-rays emitted from Ir-192, which is placed in a sealed stainless-steel capsule, is approximately $397 \mathrm{keV}$.

Figure 2 presents the experimental setup for measuring the Cerenkov radiation generated from the sensing probe of the CFOD. The sensing probe is located on the center of the Ir-192 HDR brachytherapy source. When the sensing probe is irradiated by the gamma-rays, two Cerenkov radiation signals with different light intensities are generated from the probes 1 and 2 in the sensing probe and then, they are transmitted to the pertinent channels in the PMT via two transmitting optical fibers with a length of $10 \mathrm{~m}$. The output current signals generated from the PMT are converted into the Transistor-transistor logic (TTL) pulses by a photon-counting unit. Finally, the pulse counts are transmitted through a universal serial bus (USB) line to a laptop.

\section{Results}

Figure 3 shows the light intensities of the real-time Cerenkov radiation signals generated from the sensing probe of the CFOD during the gamma-ray irradiation. The output signal of the probe 1 is higher than that of the probe

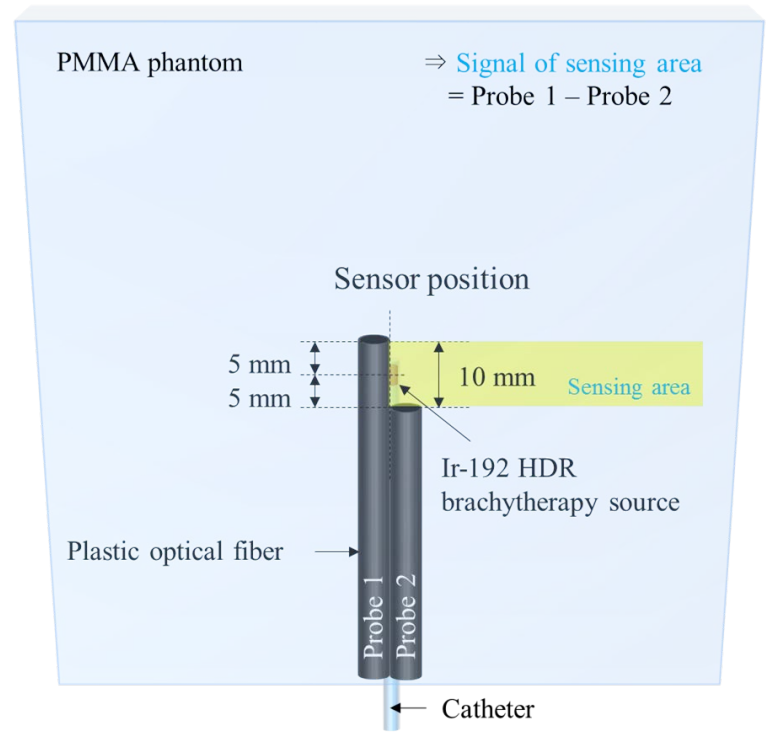

Figure 1. Schematic diagram of the sensing probe of the proposed CFOD without any scintillator. 


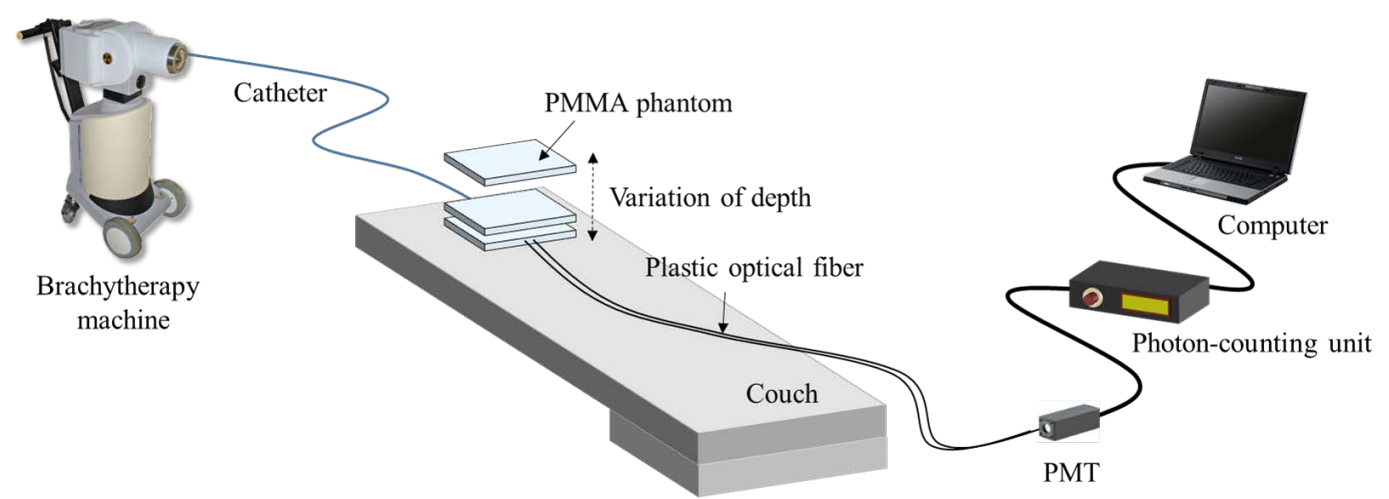

Figure 2. Experimental setup for measuring Cerenkov radiation using the CFOD and the Ir-192 HDR brachytherapy source.

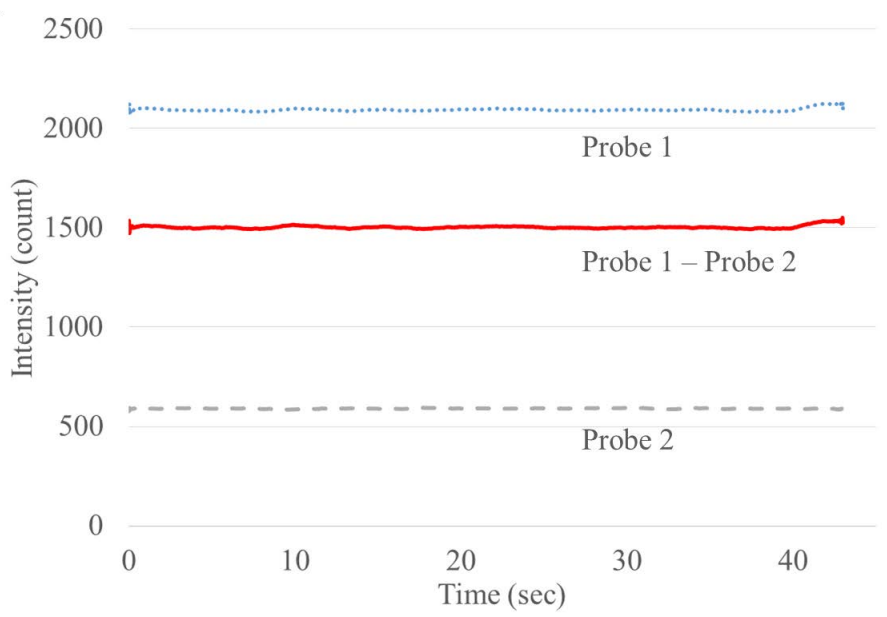

Figure 3. Subtraction method and the light intensities of the real-time Cerenkov radiation signals generated in the probes 1 and 2.

2 because the two probes have the different irradiated length. In this study, the real-time dose information is obtained by measuring the intensity difference of the two Cerenkov radiation signals from the probes 1 and 2, as shown in Figure 3 [6].

Using the subtraction method that deducts the output signal of the probe 2 from that of the probe 1 , we obtained the RDD curve according to the depth variation of the PMMA phantom, as shown in Figure 4. The RDD curve in the PMMA phantom falls off sharply to a depth of $20 \mathrm{~mm}$ and decreases gently with depth beyond 20 $\mathrm{mm}$. The RDD curve measured by using the CFOD is also compared with the simulation result of MCNPX, which is used to verify the dosimetric response of the CFOD. As experimental results, the RDD curve obtained by using the proposed CFOD is similar to the electron flux distribution calculated by using the MCNPX simulation.

\section{Conclusions}

In this study, we fabricated a CFOD using two POFs without any scintillator to measure real-time dose information for HDR brachytherapy. By using a sensing probe of the CFOD, Cerenkov radiation signals owing to the gamma-rays emitted from Ir-192 were measured successfully. We obtained the depth dose distribution using a subtraction method and then compared the RDD curve to the electron flux distribution calculated by using the MCNPX simulation.

In conclusion, we demonstrated that the proposed CFOD can be used to measure dose information including RDD distributions. Based on the results of this study, it is expected that the CFOD can give useful dose information for HDR brachytherapy due to its many advantages, including high spatial resolution, real-time monitoring, remote operation, and water or tissue-equivalence. 


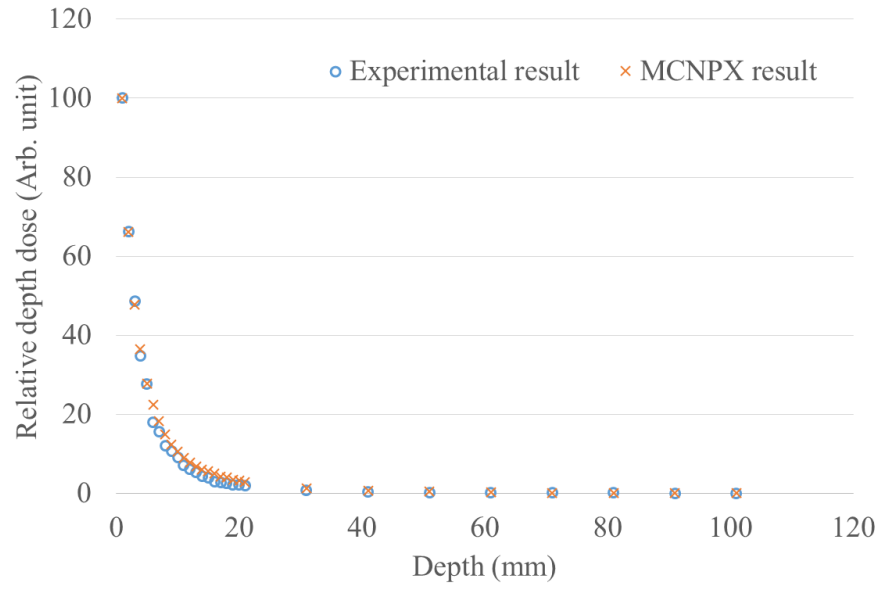

Figure 4. Comparison of two RDD curves obtained by using the CFOD and the MCNPX simulation.

\section{Acknowledgements}

This research was supported by Basic Science Research Program through the National Research Foundation of Korea (NRF) funded by the Ministry of Science, ICT and future Planning and this work was supported by the Nuclear Safety Research Program through the Korea Foundation Of Nuclear Safety (KOFONS), granted financial resource from the Nuclear Safety and Security Commission (NSSC), Republic of Korea (Nos.

2014R1A2A2A04002620, and 1403017-0114-SB110). Also, this work was supported by National Nuclear R \& D Program through the National Research Foundation of Korea (NRF) funded by the Ministry of Science, ICT and future Planning (No.2014M2B2A9031841).

\section{References}

[1] Cerenkov, P.A. (1934) Visible Glow under Exposure of Gamma Radiation. Dokl, Akad, Nauk, SSSR, 2, 451.

[2] Beddar, A.S., Kinsella, T.J., Ikhlef, A. and Sibata, C.H. (2001) A Miniature “Scintillator-Fiberoptic-PMT" Detector System for the Dosimetry of Small Fields in Stereotactic Radiosurgery. IEEE Trans. Nucl. Sci., 48, 924-928. http://dx.doi.org/10.1109/23.940133

[3] Clift, M.A., Johnston, P.N. and Webb, D.V. (2002) A Temporal Method of Avoiding the Cerenkov Radiation Generated in Organic Scintillator Dosimeters by Pulsed Mega-Voltage Electron and Photon Beams. Phys. Med. Biol., 47, 1421-1433. http://dx.doi.org/10.1088/0031-9155/47/8/313

[4] Clift, M.A., Sutton, R.A. and Webb, D.V. (2000) Dealing with Cerenkov Radiation Generated in Organic Scintillator Dosimeters by Bremsstrahlung Beams. Phys. Med. Biol., 45, 1165-1182. http://dx.doi.org/10.1088/0031-9155/45/5/307

[5] Lee, B., Jang, K.W., Cho, D. H., Yoo, W.J., Tack, G.R., Chung, S.C., Kim, S. and Cho, H. (2007) Measurements and Elimination of Cherenkov Light in Fiber-Optic Scintillating Detector for Electron Beam Therapy Dosimetry. Nucl. Instrum. Phys. Res. A, 579, 344-348. http://dx.doi.org/10.1016/j.nima.2007.04.074

[6] Frelin, A.M., Fontbonne, J.M., Ban, G., Colin, J., Labalme, M., Batalla, A., Isambert, A., Vela, A. and Leroux, T. (2005) Spectral Discrimination of Čerenkov Radiation in Scintillating Dosimeters. Med. Phys., 32, 3000-3006. http://dx.doi.org/10.1118/1.2008487

[7] Law, S.H., Fleming, S.C., Suchowerska, N. and McKenzie, D.R. (2006) Optical Fiber Design and the Trapping of Cerenkov Radiation. Appl. Opts., 45, 9151-9159. http://dx.doi.org/10.1364/AO.45.009151

[8] Law, S.H., Suchowerska, N., McKenzie, D.R., Fleming, S.C. and Lin, T. (2007) Transmission of Cerenkov Radiation in Optical Fibers. Optics Lett., 32, 1205-1207. http://dx.doi.org/10.1364/OL.32.001205

[9] Yoo, W.J., Shin, S.H., Jeon, D., Hong, S., Kim, S.G., Sim, H.I., Jang, K.W., Cho, S. and Lee, B. (2013) Simultaneous Measurements of Pure Scintillation and Cerenkov Signals in an Integrated Fiber-Optic Dosimeter for Electron Beam Therapy Dosimetry. Opt. Express, 21, 27770-27779. http://dx.doi.org/10.1364/OE.21.027770

[10] Beddar, A.S. (2006) Plastic Scintillation Dosimetry and Its Application to Radiotherapy. Radiat. Meas., 41, $124-133$. http://dx.doi.org/10.1016/j.radmeas.2007.01.002 


\section{Submit or recommend next manuscript to SCIRP and we will provide best service for you:}

Accepting pre-submission inquiries through Email, Facebook, LinkedIn, Twitter, etc.

A wide selection of journals (inclusive of 9 subjects, more than 200 journals)

Providing 24-hour high-quality service

User-friendly online submission system

Fair and swift peer-review system

Efficient typesetting and proofreading procedure

Display of the result of downloads and visits, as well as the number of cited articles

Maximum dissemination of your research work

Submit your manuscript at: http://papersubmission.scirp.org/ 